\title{
PROPER COCYCLES AND WEAK FORMS OF AMENABILITY
}

\author{
PAUL JOLISSAINT
}

\begin{abstract}
Let $G$ and $H$ be locally compact, second countable groups. Assume that $G$ acts in a measure class preserving way on a standard space $(X, \mu)$ such that $L^{\infty}(X, \mu)$ has an invariant mean and that there is a Borel cocycle $\alpha: G \times X \rightarrow H$ which is proper in the sense of 7 and 9]. We show that if $H$ has one of the three properties: Haagerup property (a-T-menability), weak amenability or weak Haagerup property, then so does $G$. In particular, we show that if $\Gamma$ and $\Delta$ are measure equivalent discrete groups in the sense of Gromov, then such cocycles exist and $\Gamma$ and $\Delta$ share the same weak amenability properties above.
\end{abstract}

\section{INTRODUCTION}

Let $G$ and $H$ be locally compact, second countable groups. If $H$ is a closed subgroup of $G$, it inherits weak amenability properties of $G$. Conversely, if in addition the homogeneous space $G / H$ is amenable in the sense that $L^{\infty}(G / H)$ has a $G$-invariant state, then $G$ in turn inherits weak amenability properties of $H$ : see for instance [1, 2], 7] and the recent article [9].

One way of proving such results is to use a natural cocycle associated to some regular Borel cross-section $\gamma: G / H \rightarrow G$ of the canonical projection $p: G \rightarrow G / H$. More precisely, we define $\alpha: G \times G / H \rightarrow H$ by

$$
\alpha(g, x)=\gamma(g x)^{-1} g \gamma(x) \quad((g, x) \in G \times G / H) .
$$

Since $g \gamma(x) H=\gamma(g x) H$ for all $g \in G$ and $x \in G / H$, we see that $\alpha(g, x) \in H$ as well.

More generally, assume that $G$ acts in a measure class preserving way on some standard probability space $(X, \mu)$. Then a Borel cocycle is a Borel map $\alpha$ : $G \times X \rightarrow H$ such that for all $g, h \in G$, one has

$$
\alpha(g h, x)=\alpha(g, h x) \alpha(h, x)
$$

for $\mu$-almost every $x \in X$. Notice that $H$ is not necessarily a closed subgroup of $G$.

The aim of the present note is to prove that weak amenability properties of $H$ are inherited by $G$ when $\alpha$ is a cocycle that satisfies a properness condition in the sense of Definition 1.3 below, and when $L^{\infty}(X)$ has a $G$-invariant state. In order to state precisely our main result, we need to recall some definitions from [2], [3], [9], 7] and [8].

Date: August 13, 2018.

2010 Mathematics Subject Classification. Primary 22D05, 22D10.

Key words and phrases. Locally compact groups, measure equivalent groups, proper cocycles, weak Haagerup property, weak amenability, Fourier algebra, Herz-Schur multipliers. 
We assume throughout the article that our groups are locally compact and second countable. The definitions of the algebras $A(G)$ and $B_{2}(G)$ are reminded in the next section.

Definition 1.1. Let $G$ be a locally compact, second countable group.

(1) (2]) We say that $G$ has the Haagerup property if there exists a sequence $\left(u_{n}\right)_{n \geq 1}$ of normalized, positive definite functions on $G$, such that $u_{n} \in$ $C_{0}(G)$ for every $n$, and that $u_{n} \rightarrow 1$ uniformly on compact subsets of $G$. $\left(C_{0}(G)\right.$ denotes the vector space of all continuous functions on $G$ that tend to 0 at infinity.)

(2) ([3]) The Cowling-Haagerup constant $\Lambda_{\mathrm{WA}}(G)$ is the infimum of all numbers $C \geq 1$ for which there exists a sequence $\left(u_{n}\right)_{n \geq 1}$ in the Fourier algebra $A(G)$ satisfying: $\left\|u_{n}\right\|_{B_{2}} \leq C$ for every $n$ and $u_{n} \rightarrow 1$ uniformly on compact sets, where $B_{2}(G)$ denotes the Herz-Schur multiplier algebra of $G$ (see [9]). Moreover, $G$ is said to be weakly amenable if $\Lambda_{\mathrm{WA}}(G)<\infty$.

(3) (9]) The weak Haagerup constant $\Lambda_{\mathrm{WH}}(G)$ is the infimum of all numbers $C \geq 1$ for which there exists a sequence $\left(u_{n}\right)_{n \geq 1} \subset B_{2}(G) \cap C_{0}(G)$ such that $\left\|u_{n}\right\|_{B_{2}} \leq C$ for every $n$ and $u_{n} \rightarrow 1$ uniformly on compact sets. Moreover, $G$ is said to have the weak Haagerup property if $\Lambda_{\mathrm{WH}}(G)<\infty$.

As $A(G) \subset B_{2}(G) \cap C_{0}(G)$, one always has $\Lambda_{\mathrm{WH}}(G) \leq \Lambda_{\mathrm{WA}}(G)$, and a weakly amenable group has the weak Haagerup property. Similarly, as normalized, positive definite functions are Herz-Schur multipliers of norm one, if $G$ has the Haagerup property then it has the weak Haagerup property and $\Lambda_{\mathrm{WH}}(G)=1$. See 9 ] for a discussion of these properties.

The following definition generalizes the notion of co-Følner groups as in [2].

Definition 1.2. ([6], $[\underline{8}$, [11]) Let $G$ be a locally compact, second countable group that acts in a measure class preserving way on the standard space $(X, \mu)$. Then we say that $(G, X)$ is an amenable pair if $L^{\infty}(X, \mu)$ has a $G$-invariant state.

Finally, let $\alpha: G \times X \rightarrow H$ be a Borel cocycle. The following is essentially taken from [7; see also 9. We need first to fix some notation: let $A \subset X$ be a Borel set and let $L$ be a compact subset of $H$; we denote by $K(A, L)$ the set of all elements $g \in G$ for which $\mu\left(X_{A}(g)\right)>0$, where

$$
X_{A}(g)=\left\{x \in A \cap g^{-1} A: \alpha(g, x) \in L\right\} .
$$

As mentioned above, it is motivated by the case where $H$ is a closed subgroup of $G$ that we present in detail now. Choose a regular Borel cross-section $\gamma: G / H \rightarrow$ $G$ for the canonical projection $p: G \rightarrow G / H$, i.e. $\gamma$ is a Borel map such that $p(\gamma(x))=x$ for every $x \in G / H$ and such that, for every compact set $K \subset G$, the set $\gamma(G / H) \cap p^{-1}(p(K))$ is precompact in $G$. Moreover, for every compact set $C \subset G / H$, there is a compact set $C_{1} \subset G$ such that $C=p\left(C_{1}\right)$. Then it is straightforward to see that $\gamma(C)$ is precompact for every compact set $C \subset G / H$.

Recall that the associated cocycle $\alpha$ is defined by

$$
\alpha(g, x)=\gamma(g x)^{-1} g \gamma(x) \quad(g \in G, x \in G / H) .
$$

Then one has:

(i) If $K \subset G$ and $A \subset G / H$ are compact sets, then $\alpha(K \times A)$ is precompact since it is contained in $\gamma(K A)^{-1} K \gamma(A)$. 
(ii) If $A \subset G / H$ and $L \subset H$ are compact, it is easy to see that, if $g \in G$ is such that $X_{A}(g) \neq \emptyset$, then $g \in \gamma(A) L \gamma(A)^{-1}$. In particular $K(A, L)$ is precompact.

As $G$ and $G / H$ are in particular $\sigma$-compact, we see that the cocycle $\alpha$ satisfies the next definition.

Definition 1.3. The cocycle $\alpha: G \times X \rightarrow H$ is proper if it satisfies the following two conditions:

(i) for every Borel set $A \subset X$, for every $\varepsilon>0$ and for every compact set $K \subset G$, there exists a Borel set $A_{\varepsilon, K} \subset A$ such that $\mu\left(A \backslash A_{\varepsilon, K}\right)<\varepsilon$ and $\alpha\left(K \times A_{\varepsilon, K}\right)$ is precompact.

(ii) For every compact set $L \subset H$ and every $\varepsilon>0$, there exists a Borel set $A \subset X$ such that $\mu(X \backslash A) \leq \varepsilon$ and $K(A, L)$ is precompact.

We observe first that properness of $\alpha$ is independent of the chosen probability measure.

Lemma 1.4. Let $\alpha: G \times X \rightarrow H$ be a proper cocycle with respect to the probability measure $\mu$ on $X$, and let $\nu$ be an equivalent probability measure on $X$. Then $\alpha$ is proper with respect to $\nu$.

Proof. This follows immediately from Theorem 6.11 of [10]: as $\nu$ is equivalent to $\mu$, it follows in particular that for every $\varepsilon>0$, there exists $\theta>0$ such that, if $B \subset X$ is Borel and if $\mu(B) \leq \theta$, then $\nu(B) \leq \varepsilon$.

Before giving examples of such cocycles, let us state our first main result:

Theorem 1.5. Let $G$ and $H$ be locally compact, second countable groups, let $G$ act on some probability space $(X, \mu)$ so that $(G, X)$ is an amenable pair, and let $\alpha: G \times X \rightarrow H$ be a proper cocycle.

(a) If $H$ has the Haagerup property, then so does $G$.

(b) If $H$ is weakly amenable group then so is $G$, and

$$
\Lambda_{\mathrm{WA}}(G) \leq \Lambda_{\mathrm{WA}}(H) .
$$

(c) If $H$ has the weak Haagerup property, then so does $G$, and

$$
\Lambda_{\mathrm{WH}}(G) \leq \Lambda_{\mathrm{WH}}(H) .
$$

As we will see, the proofs of the three statements rely on the same techniques, and they will be given in the next section.

As promised, here are examples of proper cocycles.

Example 1.6. Every cocycle $\alpha$ described below is proper.

(1) Let $G$ and $H$ be locally compact, second countable groups and assume that $\sigma: G \rightarrow H$ is a continuous homomorphism with compact kernel, and let $(X, \mu)$ be an arbitrary standard probability $G$-space. Define $\alpha: G \times X \rightarrow H$ by

$$
\alpha(g, x)=\sigma(g) \quad((g, x) \in G \times X) .
$$

(2) More generally, let $G$ and $H$ be as in Example (1), let $G_{0}$ be a closed subgroup of $G$ and assume that $\sigma: G_{0} \rightarrow H$ is a continuous homomorphism with compact kernel. Choose a regular Borel cross-section $\gamma: G / G_{0} \rightarrow G$ for the canonical projection and a quasi-invariant probability measure $\nu$ 
on $G / G_{0}$. If $(Y, \mu)$ is an arbitrary standard probability $G$-space, equip $\left(G / G_{0} \times Y, \nu \times \mu\right)$ with the product action and define $\alpha: G \times\left(G / G_{0} \times Y\right) \rightarrow$ $H$ by

$$
\alpha(g,(x, y))=\sigma\left(\gamma(g x)^{-1} g \gamma(x)\right) .
$$

This case generalizes the situation where $H=G_{0}$ is a closed subgroup of $G$.

(3) Let $\pi: P \rightarrow B$ be a (metrizable, locally compact) topological principal fiber bundle with countable structure group $H$, and assume that a locally compact, second countable group $G$ acts continuously on $P$ so that the $G$-action commutes with the $H$-action. Using a bounded measurable cross-section for $\pi$, we get a trivialization of $P \cong H \times B$ which preserves precompact subsets. Then the corresponding action of $G$ on $H \times B$ is given by

$$
g \cdot(h, x)=(\alpha(g, x) h, g x)
$$

where $\alpha$ is a Borel cocycle. If the action of $G$ on $P$ is proper, then $\alpha$ is a proper cocycle.

As will be proved in the last section, an important fourth family of pairs of groups that give rise to proper cocycles is the family of pairs of countable, discrete groups $\Gamma$ and $\Delta$ that satisfy Gromov's notion of measure equivalence. We recall the latter from [4]:

Definition 1.7. We say that $\Gamma$ and $\Delta$ are measure equivalent if there exist commuting, measure-preserving, free actions of $\Gamma$ and $\Delta$ on some infinite-measure standard space $(\Sigma, \sigma)$, such that $\Gamma$ and $\Delta$ both admit fundamental domains with finite measure. (For convenience, we denote the action of $\Gamma$ on the left and the action of $\Delta$ on the right.)

Measure equivalence is a weak form of orbit equivalence: see [5], Section 3. Let us generalize it slightly as follows.

Definition 1.8. We say that $\Gamma$ and $\Delta$ are amenably measure equivalent if there exist commuting, measure-preserving, free actions of $\Gamma$ and $\Delta$ on some infinitemeasure standard space $(\Sigma, \sigma)$, such that the pairs $(\Gamma, \Sigma / \Delta)$ and $(\Delta, \Gamma \backslash \Sigma)$ are both amenable pairs.

Example 1.9. Let $\Gamma$ and $\Delta$ be discrete subgroups of the same locally compact, second countable, unimodular group $G$ such that the homogeneous spaces $G / \Gamma$ and $G / \Delta$ both have $G$-invariant means, i.e. the pairs $(G, G / \Gamma)$ and $(G, G / \Delta)$ are amenable. Then $\Gamma$ and $\Delta$ are amenably measure equivalent groups.

As claimed above, amenably measure equivalent groups give rise to proper cocycles, so that Theorem 1.5 will be used to prove our last result.

Theorem 1.10. Let $\Gamma$ and $\Delta$ be amenably measure equivalent groups.

(1) If one of them has the Haagerup property, then the other one has the same property.

(2) The following equalities hold:

$$
\Lambda_{\mathrm{WA}}(\Gamma)=\Lambda_{\mathrm{WA}}(\Delta) \quad \text { and } \quad \Lambda_{\mathrm{WH}}(\Gamma)=\Lambda_{\mathrm{WH}}(\Delta) .
$$

Let us discuss instances where Theorem 1.10 applies in the context of semidirect products. Let $A$ be an amenable, countable group. If it acts on some group $\Gamma$, then it is easy to see that $\Gamma$ and $\Gamma \rtimes A$ are amenably measure equivalent. 
The fact that $\Gamma$ and $\Gamma \rtimes A$ have simultaneously the Haagerup property and that their constants $\Lambda_{\mathrm{WA}}$ and $\Lambda_{\mathrm{WH}}$ coincide is already known: it follows respectively from [2], [7] and [9].

Next, let us assume that the amenable group $A$ is on the other side: let $\theta: \Delta \rightarrow$ $\operatorname{Aut}(A)$ be an action of the countable group $\Delta$ on $A$, and consider the following action of the semidirect product group $\Gamma:=A \rtimes_{\theta} \Delta$ on $A$ :

$$
(a, \delta) \cdot b=a \theta_{\delta}(b)
$$

for all $a, b \in A$ and all $\delta \in \Delta$. Then we have:

Proposition 1.11. Let $A, \Delta$ and $\theta$ be as above and assume that there exists a sequence $\left(F_{k}\right)_{k \geq 1}$ of finite subsets of $A$ such that, for all $(a, \delta) \in \Gamma$

$$
\frac{\left|a \theta_{\delta}\left(F_{k}\right) \triangle F_{k}\right|}{\left|F_{k}\right|} \rightarrow 0
$$

as $k \rightarrow \infty$. In other words, the pair $(\Gamma, A)$ is amenable in the sense of Definition 1.2. Then $\Gamma$ and $\Delta$ are amenably measure equivalent.

Proof. Take $\Sigma=A \times \Delta$ with counting measure. The action (on the left) of $\Gamma=A \rtimes \Delta$ is defined by multiplication in the semidirect product, namely

$$
(a, \delta) \cdot\left(b, \delta^{\prime}\right)=\left(a \theta_{\delta}(b), \delta \delta^{\prime}\right)
$$

and the action of $\Delta$ (on the right) is given by $(a, \delta) \cdot \delta^{\prime}=\left(a, \delta \delta^{\prime}\right)$. Then $\Gamma \backslash \Sigma$ is the one-point space and $\Sigma / \Delta$ is isomorphic to $A$ as a $\Gamma$-space. The amenability of the pair $(\Gamma, A)$ implies that $\Gamma$ and $\Delta$ are amenably measure equivalent.

Let us illustrate Proposition 1.11 by an example.

Example 1.12. Let $1 \rightarrow Z \rightarrow A \rightarrow Q \rightarrow 1$ be a central extension of some amenable group $Q$. Denote by $s: Q \rightarrow A$ a section of the canonical projection with $s(1)=1$.

Let also $h: \Delta \rightarrow Q$ be a homomorphism of some group $\Delta$. With these data, define an action $\theta$ of $\Delta$ on $A$ by

$$
\theta_{\delta}=\operatorname{Ad}(s \circ h(\delta)) \quad \forall \delta \in \Delta .
$$

As each automorphism $\theta_{\delta}$ is inner, $(A, \Delta, \theta)$ fulfills the conditions of Proposition 1.11 indeed, since $A$ is amenable, the direct product group $A \times A$ is as well, and the action $(a, b) \cdot x=a x b^{-1}$ of $A \times A$ on $A$ admits a sequence $\left(F_{k}\right)_{k \geq 1}$ of finite subsets of $A$ such that

$$
\frac{\left|(a, b) \cdot F_{k} \triangle F_{k}\right|}{\left|F_{k}\right|} \rightarrow 0
$$

as $k \rightarrow \infty$ for all $a, b \in A$.

Remark also that $s \circ h$ is not a homomorphism in general, thus $\Gamma:=A \rtimes_{\theta} \Delta$ is not isomorphic to the direct product $A \times \Delta$.

Acknowledgements. I am very grateful to Tadeusz Januszkiewicz and the referee for their careful reading of the manuscript and their valuable comments. 


\section{Proof of Theorem 1.5}

Let $G$ and $H$ be locally compact groups; we assume that they are second countable even if definitions below make sense for arbitrary locally compact groups.

The Fourier-Stieltjes algebra of $G$ is the set of all coefficient functions of unitary representations of $G$, thus, for every $u \in B(G)$ there exists a unitary representation $(\pi, \mathcal{H})$ of $G$ and two vectors $\xi, \eta \in \mathcal{H}$ such that $u(g)=\langle\pi(g) \xi \mid \eta\rangle$ for every $g \in G$. It is a Banach algebra with respect to the norm

$$
\|u\|_{B}=\inf \|\xi\|\|\eta\|
$$

where the infimum is taken over all representations of $u$ as above.

The Fourier algebra of $G$ is the set of all coefficient functions associated to the left regular representation $\lambda$ of $G$ (which acts on $L^{2}(G)$ ). It is the norm closure of the algebra of compactly supported continuous functions $C_{c}(G) \cap B(G)$ in the algebra $B(G)$.

A Herz-Schur multiplier of $G$ is a continous function $u: G \rightarrow \mathbb{C}$ for which there exists a separable Hilbert space $\mathcal{H}$ and two bounded, continuous functions $\xi, \eta: G \rightarrow \mathcal{H}$ such that

$$
u\left(h^{-1} g\right)=\langle\xi(g) \mid \eta(h)\rangle \quad(g, h \in G) .
$$

It turns out that the set $B_{2}(G)$ of all Herz-Schur multipliers on $G$ is a Banach algebra with respect to the pointwise product and to the norm

$$
\|u\|_{B_{2}}=\inf \|\xi\|_{\infty}\|\eta\|_{\infty}
$$

where the infimum is taken over all representations of $u$ as above.

Assume from now on that $G$ acts in a measure class preserving way on a standard probability space $(X, \mu)$ and that $\alpha: G \times X \rightarrow H$ is a (not necessarily proper) Borel cocycle.

We denote by $(g, x) \mapsto \chi(g, x)$ the Radon-Nikodym derivative related to the action of $G$ on $X$ and characterized by

$$
\int_{X} f(g x) \chi(g, x) d \mu(x)=\int_{X} f(x) d \mu(x) \quad \forall f \in L^{1}(X, \mu) .
$$

It satisfies the cocycle relation:

$$
\chi(g h, x)=\chi(g, h x) \chi(h, x)
$$

for all $g, h \in G$ and $\mu$-a.e. $x \in X$. Taking $f=1_{X}$, we have: $\int_{X} \chi(g, x) d \mu(x)=1$ for every $g \in G$.

For future use, let us observe that for every $g \in G$ and every Borel set $B \subset X$, one has, by Cauchy-Schwarz Inequality:

$$
\int_{B} \sqrt{\chi(g, x)} d \mu(x) \leq \mu(B)^{1 / 2}\left(\int_{X} \chi(g, x) d \mu(x)\right)^{1 / 2}=\mu(B)^{1 / 2}
$$

and

$$
\int_{g^{-1} B} \sqrt{\chi(g, x)} d \mu(x) \leq \mu(X)^{1 / 2}\left(\int_{X} 1_{B}(g x) \chi(g, x) d \mu(x)\right)^{1 / 2}=\mu(B)^{1 / 2} .
$$


The proof of Theorem 1.5 relies on two auxiliary results.

Lemma 2.1. Let $u \in L^{\infty}(H)$. Define $\hat{u}: G \rightarrow \mathbb{C}$ by

$$
\hat{u}(g)=\int_{X} u(\alpha(g, x)) \sqrt{\chi(g, x)} d \mu(x) \quad \forall g \in G .
$$

(a) If $u \in B_{2}(H)$ is a Herz-Schur multiplier on $H$, then $\hat{u} \in B_{2}(G)$ and $\|\hat{u}\|_{B_{2}} \leq$ $\|u\|_{B_{2}}$. If furthermore $u$ is positive definite, so is $\hat{u}$.

(b) If $\alpha$ is proper and if $u \in C_{0}(H)$ then $\hat{u} \in C_{0}(G)$. In particular, if $u \in$ $B_{2}(H) \cap C_{0}(H)$, then $\hat{u} \in B_{2}(G) \cap C_{0}(G)$.

(c) If $\alpha$ is proper and if $u \in A(H)$, then $\hat{u} \in A(G)$.

Proof. (a) There exist a separable Hilbert space $\mathcal{H}$ and bounded, continuous functions $\xi, \eta: H \rightarrow \mathcal{H}$ such that

(1) $u\left(t^{-1} s\right)=\langle\xi(s) \mid \eta(t)\rangle$ for all $s, t \in H$;

(2) $\|u\|_{B_{2}} \leq\|\xi\|_{\infty}\|\eta\|_{\infty}$.

Define $\hat{\xi}, \hat{\eta}: G \rightarrow L^{2}(X, \mu, \mathcal{H})$ by

$$
\hat{\xi}(g)(x)=\xi\left(\alpha\left(g^{-1}, x\right)^{-1}\right) \sqrt{\chi\left(g^{-1}, x\right)}
$$

and

$$
\hat{\eta}(g)(x)=\eta\left(\alpha\left(g^{-1}, x\right)^{-1}\right) \sqrt{\chi\left(g^{-1}, x\right)} .
$$

One has for every $g \in G$ :

$$
\begin{aligned}
\|\hat{\xi}(g)\|^{2} & =\int_{X}\left\|\xi\left(\alpha\left(g^{-1}, x\right)\right)\right\|^{2} \chi\left(g^{-1}, x\right) d \mu(x) \\
& \leq\|\xi\|_{\infty}^{2} \int_{X} \chi\left(g^{-1}, x\right) d \mu(x)=\|\xi\|_{\infty}^{2} .
\end{aligned}
$$

Similarly, $\|\hat{\eta}\|_{\infty} \leq\|\eta\|_{\infty}$. We are going to prove that, for all $g, h \in G$, one has:

$$
\hat{u}\left(h^{-1} g\right)=\langle\hat{\xi}(g) \mid \hat{\eta}(h)\rangle .
$$

It turns out that $\hat{u}$ is a continuous function on $G$ by Appendix $\mathrm{C}$ of [9] (even though $\hat{\xi}$ and $\hat{\eta}$ are not necessarily continuous).

Observe that the cocycle relation $\alpha(g h, x)=\alpha(g, h x) \alpha(h, x)$ for all $g, h \in G$ and for $\mu$-a.e $x \in X$ implies that

$$
\alpha\left(h^{-1} g, g^{-1} x\right) \alpha\left(g^{-1}, x\right)=\alpha\left(h^{-1}, x\right)
$$

and similarly

$$
\chi\left(h^{-1} g, g^{-1} x\right) \chi\left(g^{-1}, x\right)=\chi\left(h^{-1}, x\right)
$$


for all $g, h \in G$ and $\mu$-a.e. $x \in X$. Fix $g, h \in G$. One has:

$$
\begin{aligned}
\langle\hat{\xi}(g) \mid \hat{\eta}(h)\rangle & =\int_{X}\left\langle\xi\left(\alpha\left(g^{-1}, x\right)^{-1}\right) \mid \eta\left(\alpha\left(h^{-1}, x\right)^{-1}\right)\right\rangle \sqrt{\chi\left(g^{-1}, x\right) \chi\left(h^{-1}, x\right)} d \mu(x) \\
& =\int_{X} u\left(\alpha\left(h^{-1}, x\right) \alpha\left(g^{-1}, x\right)^{-1}\right) \sqrt{\chi\left(g^{-1}, x\right) \chi\left(h^{-1}, x\right)} d \mu(x) \\
& =\int_{X} u\left(\alpha\left(h^{-1} g, g^{-1} x\right)\right) \sqrt{\chi\left(g^{-1}, x\right) \chi\left(h^{-1}, x\right)} d \mu(x) \\
& =\int_{X} u\left(\alpha\left(h^{-1} g, g^{-1} x\right)\right) \chi\left(g^{-1}, x\right) \sqrt{\chi\left(h^{-1} g, g^{-1} x\right)} d \mu(x) \\
& =\int_{X} u\left(\alpha\left(h^{-1} g, x\right)\right) \sqrt{\chi\left(h^{-1} g, x\right)} d \mu(x)=\hat{u}\left(h^{-1} g\right) .
\end{aligned}
$$

If furthermore $u$ is positive definite, then one can take $\eta=\xi$, and it is straightforward to see that $\hat{u}$ is positive definite on $G$ as well. (In fact, let $\left(\pi_{u}, \mathcal{H}_{u}, \xi_{u}\right)$ be the Gel'fand-Naimark-Segal triple associated to $u$. Then, as $u(h)=\left\langle\pi_{u}(h) \xi_{u} \mid \xi_{u}\right\rangle$ for every $h \in H$, we see that the function $h \mapsto \xi(h)=\pi_{u}(h) \xi_{u}$ works.)

(b) Let now $u \in C_{0}(H)$. We assume without loss of generality that $\|u\|_{\infty} \leq 1$.

Fix $\varepsilon>0$. There exists a compact set $L \subset H$ such that $|u(h)| \leq \frac{\varepsilon}{2}$ for all $h \notin L$. Choose next a Borel set $A \subset X$ which satisfies: $\mu(X \backslash A) \leq \frac{\varepsilon^{2}}{16}$ and for which $K=\overline{K(A, L)}$ is compact.

Fix $g \in G \backslash K$. One has:

$$
\begin{aligned}
|\hat{u}(g)| \leq & \int_{\{\alpha(g, x) \in L\}}|u(\alpha(g, x))| \sqrt{\chi(g, x)} d \mu(x) \\
& +\int_{\{\alpha(g, x) \notin L\}}|u(\alpha(g, x))| \sqrt{\chi(g, x)} d \mu(x) \\
\leq & \int_{X \backslash\left(A \cap g^{-1} A\right)}|u(\alpha(g, x))| \sqrt{\chi(g, x)} d \mu(x)+\frac{\varepsilon}{2} \\
\leq & \int_{X \backslash A} \sqrt{\chi(g, x)} d \mu(x)+\int_{g^{-1}(X \backslash A)} \sqrt{\chi(g, x)} d \mu(x)+\frac{\varepsilon}{2} \\
\leq & 2 \mu(X \backslash A)^{1 / 2}+\frac{\varepsilon}{2} \leq \varepsilon .
\end{aligned}
$$

(c) The assertion is similar to that contained in Proposition 2.8 of [7, but we give a proof for the sake of completeness. We have to prove that $\hat{u}$ is in the norm closure of $C_{c}(G) \cap B(G)$ in the Fourier-Stieltjes algebra $B(G)$. Thus, let us assume that $u(h)=\langle\lambda(h) \xi \mid \eta\rangle$ with $\xi, \eta \in C_{c}(H)$. Then it is straighforward to check that

$$
\hat{u}(g)=\left\langle\lambda_{\alpha}(g) 1_{X} \otimes \xi \mid 1_{X} \otimes \eta\right\rangle \quad(g \in G)
$$

where $\lambda_{\alpha}$ is the unitary representation of $G$ on $L^{2}\left(X, \mu, L^{2}(H)\right)$ defined by

$$
\left(\lambda_{\alpha}(g) \zeta\right)(x)=\lambda\left(\alpha\left(g^{-1}, x\right)^{-1}\right) \zeta\left(g^{-1} x\right) \sqrt{\chi\left(g^{-1}, x\right)}
$$


for $\zeta \in L^{2}\left(X, \mu, L^{2}(H)\right)$. Hence $\hat{u}$ is a norm limit in $B(G)$ of functions of the form

$$
\hat{u}_{A}(g)=\left\langle\lambda_{\alpha}(g) 1_{A} \otimes \xi \mid 1_{A} \otimes \eta\right\rangle
$$

with $A \subset X$ Borel. Denote by $L$ the support of $u$. For every Borel set $A \subset X$ such that $K(A, L)$ is precompact, we have

$$
\hat{u}_{A}(g)=\int_{A \cap g^{-1} A} u(\alpha(g, x)) \sqrt{\chi(g, x)} d \mu(x) \quad(g \in G) .
$$

If $g \notin \overline{K(A, L)}$, the latter being compact in $G$, then $\mu\left(\left\{x \in A \cap g^{-1} A: \alpha(g, x) \in\right.\right.$ $L\})=0$ by Definition 1.3 and this implies that $\hat{u}_{A}(g)=0$.

Assume now that $(G, X)$ is an amenable pair. Denote by $\beta$ the action of $G$ on $L^{1}(X, \mu)$ given by

$$
\beta_{g}(f)(x)=f\left(g^{-1} x\right) \chi\left(g^{-1}, x\right)
$$

Since the set of normal states is weak* dense in the set of all states of $L^{\infty}(X)$, the amenability of $(G, X)$ is equivalent to the existence of a sequence $\left(f_{n}\right) \subset L^{1}(X, \mu)$ such that

(1) $f_{n} \geq 0$ and $\left\|f_{n}\right\|_{1}=\int_{X} f_{n}(x) d \mu(x)=1$ for every $n$;

(2) for every compact set $K \subset G$,

$$
\sup _{g \in K}\left\|\beta_{g}\left(f_{n}\right)-f_{n}\right\|_{1} \rightarrow 0
$$

as $n \rightarrow \infty$.

Lemma 2.2. If the pair $(G, X)$ is amenable, then there exists a sequence of probability measures $\left(\mu_{n}\right)$ on $X$ such that:

(a) $\mu_{n}$ is equivalent to $\mu$ for every $n$;

(b) for every compact set $K \subset G$,

$$
\sup _{g \in K} \int_{X}\left|\sqrt{\chi_{n}(g, x)}-1\right| d \mu_{n}(x) \rightarrow 0
$$

as $n \rightarrow \infty$, where $\chi_{n}(g, \cdot)=d g_{*}^{-1} \mu_{n} / d \mu_{n}$ denotes the corresponding RadonNikodym derivative.

Proof. Let $\left(f_{n}\right)$ be a sequence in $L^{1}(X, \mu)$ as above. Adding the constant function $\frac{1}{n} 1_{X}$ to $f_{n}$ and renormalizing if necessary, we assume that $\left(f_{n}\right)$ satisfies conditions (1) and (2) above, and that there exists a constant $c_{n}>0$ for every $n$ such that $f_{n} \geq c_{n}$ for every $n$. Define $\mu_{n}$ by

$$
\int_{X} f(x) d \mu_{n}(x)=\int_{X} f(x) f_{n}(x) d \mu(x) .
$$


PAUL JOLISSAINT

Then $L^{\infty}\left(X, \mu_{n}\right)=L^{\infty}(X, \mu)$ isometrically, and we have for $f \in L^{\infty}(X, \mu), g \in G$ and $n \geq 1$ :

$$
\begin{aligned}
\left|\int_{X} f(x)\left\{\chi_{n}(g, x)-1\right\} d \mu_{n}(x)\right| & =\left|\int_{X} f\left(g^{-1} x\right) f_{n}(x) d \mu(x)-\int_{X} f(x) f_{n}(x) d \mu(x)\right| \\
& =\left|\int_{X} f(x)\left\{f_{n}(g x) \chi(g, x)-f_{n}(x)\right\} d \mu(x)\right| \\
& =\left|\int_{X} f(x)\left\{\beta_{g^{-1}}\left(f_{n}\right)(x)-f_{n}(x)\right\} d \mu(x)\right| \\
& \leq\|f\|_{\infty}\left\|\beta_{g^{-1}}\left(f_{n}\right)-f_{n}\right\|_{1} .
\end{aligned}
$$

This implies that

$$
\left\|\chi_{n}(g, \cdot)-1\right\|_{L^{1}\left(X, \mu_{n}\right)}=\int_{X}\left|\chi_{n}(g, x)-1\right| d \mu_{n}(x) \leq\left\|\beta_{g^{-1}}\left(f_{n}\right)-f_{n}\right\|_{L^{1}(X, \mu)}
$$

for every $g \in G$ and every $n$. Let $K$ be a compact subset of $G$ and $\varepsilon>0$. There exists $N>0$ such that

$$
\sup _{g \in K}\left\|\beta_{g^{-1}}\left(f_{n}\right)-f_{n}\right\|_{L^{1}(X, \mu)} \leq \varepsilon
$$

for every $n \geq N$, so that we get for $g \in K$ and $n \geq N$ :

$$
\begin{aligned}
\int_{X}\left|\sqrt{\chi_{n}(g, x)}-1\right| d \mu_{n}(x) & \leq \int_{X}\left|\sqrt{\chi_{n}(g, x)}-1\right|\left|\sqrt{\chi_{n}(g, x)}+1\right| d \mu_{n}(x) \\
& =\int_{X}\left|\chi_{n}(g, x)-1\right| d \mu_{n}(x) \leq \varepsilon .
\end{aligned}
$$

Proof of Theorem 1.5. We are going to prove statement (c) of Theorem 1.4. The proofs of (a) and (b) are special cases that will be discussed briefly afterwards.

Thus let us assume that $H$ has the weak Haagerup property.

Fix $C>\Lambda_{\mathrm{WH}}(H), K \subset G$ compact and $\varepsilon>0$. We are going to prove that there exists $\hat{u} \in B_{2}(G) \cap C_{0}(G)$ such that:

(1) $\|\hat{u}\|_{B_{2}} \leq C$;

(2) $\sup _{g \in K}|\hat{u}(g)-1| \leq \varepsilon$.

Let $\left(\mu_{n}\right)$ be as in Lemma 2.2, and let $n$ be large enough in order that

$$
\sup _{g \in K} \int_{X}\left|\sqrt{\chi_{n}(g, x)}-1\right| d \mu_{n}(x) \leq \frac{\varepsilon}{4(C+1)}=: \varepsilon^{\prime} .
$$

By condition (i) of Definition 1.3 and Lemma 1.4, there exists a Borel set $A=A_{\varepsilon^{\prime}, K}$ such that $\alpha(K \times A)$ is precompact and

$$
\mu_{n}(X \backslash A) \leq \frac{\varepsilon}{4(C+1)} .
$$


Let $L$ denote the closure of $\alpha(K \times A)$ in $H$ and choose $u \in B_{2}(H) \cap C_{0}(H)$ such that

and put

$$
\|u\|_{B_{2}} \leq C \quad \text { and } \quad \sup _{h \in L}|u(h)-1| \leq \frac{\varepsilon}{4}
$$

$$
\hat{u}(g)=\int_{X} u(\alpha(g, x)) \sqrt{\chi_{n}(g, x)} d \mu_{n}(x)
$$

for $g \in G$. Lemma 2.1 implies that $\hat{u} \in B_{2}(G) \cap C_{0}(G)$ and that $\|\hat{u}\|_{B_{2}} \leq C$.

We have for every $g \in K$ :

$$
\begin{aligned}
|\hat{u}(g)-1| \leq & \int_{A}\left|u(\alpha(g, x)) \sqrt{\chi_{n}(g, x)}-1\right| d \mu_{n}(x) \\
& +\int_{A^{c}}\left|u(\alpha(g, x)) \sqrt{\chi_{n}(g, x)}-1\right| d \mu_{n}(x) .
\end{aligned}
$$

Then

$$
\begin{aligned}
\int_{A}\left|u(\alpha(g, x)) \sqrt{\chi_{n}(g, x)}-1\right| d \mu_{n}(x) \leq & \int_{A}|u(\alpha(g, x))|\left|\sqrt{\chi_{n}(g, x)}-1\right| d \mu_{n}(x) \\
& +\int_{A}|u(\alpha(g, x))-1| d \mu_{n}(x) \\
\leq & C \cdot \int_{X}\left|\sqrt{\chi_{n}(g, x)}-1\right| d \mu_{n}(x)+\frac{\varepsilon}{4} \\
\leq & \frac{\varepsilon}{2} .
\end{aligned}
$$

Next,

$$
\begin{aligned}
& \int_{A^{c}}\left|u(\alpha(g, x)) \sqrt{\chi_{n}(g, x)}-1\right| d \mu_{n}(x) \\
& \leq \int_{A^{c}}|u(\alpha(g, x))|\left|\sqrt{\chi_{n}(g, x)}-1\right| d \mu_{n}(x) \\
& \quad+\int_{A^{c}}|u(\alpha(g, x))-1| d \mu_{n}(x) \\
& \leq \quad C \cdot \int_{X}\left|\sqrt{\chi_{n}(g, x)}-1\right| d \mu_{n}(x) \\
& \quad+(C+1) \mu_{n}(X \backslash X(K, L)) \\
& \leq \frac{\varepsilon}{2}
\end{aligned}
$$

This ends the proof of statement (c).

If $H$ satisfies condition (a), given $K \subset G$ compact and $\varepsilon>0$, the same construction as above from a positive definite, normalized function $u \in C_{0}(H)$ gives a positive definite function $\hat{u} \in C_{0}(G)$ that satisfies

$$
\sup _{g \in K}|\hat{u}(g)-1| \leq \varepsilon
$$


This proves that $G$ has the Haagerup property.

Finally, if $H$ satisfies condition (b), if $C>\Lambda_{\mathrm{WA}}(H), K \subset G$ compact and $\varepsilon>0$ are given, choosing $u \in A(H)$ with $\|u\|_{B_{2}} \leq C$ as in the first part of the proof, we get $\hat{u} \in A(G)$ satisfying (1) and (2) above. This proves that $G$ is weakly amenable and that $\Lambda_{\mathrm{WA}}(G) \leq \Lambda_{\mathrm{WA}}(H)$.

\section{Proof of theorem 1.10}

Let $\Gamma$ and $\Delta$ be amenably measure equivalent groups as in Theorem 1.10 and let $(\Sigma, \sigma)$ be a standard infinite measure space on which $\Gamma$ and $\Delta$ act, the first one acting freely on the left and the second one freely on the right, both actions preserving the infinite measure $\sigma$, and such that the pairs $(\Gamma, \Sigma / \Delta)$ and $(\Delta, \Gamma \backslash \Sigma)$ are amenable.

We fix our notation and recall some needed facts from 4]: we choose Borel cross-sections from $\Gamma \backslash \Sigma$ and $\Sigma / \Delta$ to $\Sigma$, and we denote by $Y$ and $X$ their respective ranges so that

$$
\Sigma=\bigsqcup_{\delta \in \Delta} X \delta=\bigsqcup_{\gamma \in \Gamma} \gamma Y
$$

and that they are standard measure spaces endowed with the corresponding restrictions of $\sigma$, say $\mu=\left.\sigma\right|_{X}$ and $\nu=\left.\sigma\right|_{Y}$. The action of $\Gamma$ on $\Sigma / \Delta$ is isomorphic to the following action of $\Gamma$ on $X$ : for each pair $(\gamma, x) \in \Gamma \times X$, there exists a unique element $\alpha(\gamma, x) \in \Delta$ such that $\gamma x \in X \alpha(\gamma, x)$. Hence the element $\gamma \cdot x:=\gamma x \alpha(\gamma, x)^{-1}$ belongs to $X$, and the mapping $(\gamma, x) \mapsto \gamma \cdot x$ defines an action of $\Gamma$ on $X$, and $\alpha$ is a Borel cocycle with values in $\Delta$ :

$$
\alpha\left(\gamma_{1} \gamma_{2}, x\right)=\alpha\left(\gamma_{1}, \gamma_{2} \cdot x\right) \alpha\left(\gamma_{2}, x\right) \quad \forall x \in X, \forall \gamma_{1}, \gamma_{2} \in \Gamma .
$$

Similarly, the action of $\Delta$ on the orbit space $\Gamma \backslash \Sigma$ is isomorphic to the following action of $\Delta$ on $Y$ : for each pair $(y, \delta) \in Y \times \Delta$ there exists a unique $\beta(y, \delta) \in \Gamma$ such that $y \delta \in \beta(y, \delta) Y$. Then set $y \cdot \delta=\beta(y, \delta)^{-1} y \delta \in Y$, so that this defines an action of $\Delta$ on $Y$ on the right, and $\beta$ is a Borel cocycle for this action, viz

$$
\beta\left(y, \delta_{1}\right) \beta\left(y \cdot \delta_{1}, \delta_{2}\right)=\beta\left(y, \delta_{1} \delta_{2}\right) \quad \forall y \in Y, \forall \delta_{1}, \delta_{2} \in \Delta .
$$

Observe that $\mu$ (resp. $\nu$ ) is $\Gamma$-invariant (resp. $\Delta$-invariant), but that it need not be finite. However, there are invariant states on $L^{\infty}(X)$ and on $L^{\infty}(Y)$.

Theorem 1.10 will be a straightforward consequence of Theorem 1.5 for two reasons: the cocycle $\alpha$ is proper as the following lemma shows, and amenably measure equivalence is a symmetric property.

Lemma 3.1. Retaining notation above, $\alpha$ is a proper cocycle from $\Gamma \times X$ to $\Delta$. More precisely:

(a) Let $1 \in K \subset \Gamma$ be finite, let $A \subset X$ be a Borel set with finite measure and let $\varepsilon>0$. Then there exists a Borel set $A_{\varepsilon, K} \subset A$ and a finite set $F \subset \Delta$ such that $\sigma\left(A \backslash A_{\varepsilon, K}\right)<\varepsilon$ and

$$
\bigcup_{\gamma \in K} \gamma A_{\varepsilon, K} \subset \bigsqcup_{\delta \in F} X \delta
$$

In particular $\alpha\left(K \times A_{\varepsilon, K}\right)$ is a finite set and $\alpha$ satisfies condition (i) of Definition 1.3. 
(b) Let $1 \in F \subset \Delta$ be a finite set and let $\mathcal{A}_{F}$ be the set of Borel subsets $A$ of $X$ with finite measure for which there exists a finite set $K=K(A, F) \subset \Gamma$ such that

$$
\bigsqcup_{\delta \in F} A \delta \subset \bigsqcup_{\gamma \in K} \gamma Y
$$

Then the elements of $\mathcal{A}_{F}$ have the following properties:

(b1) For every Borel set $A \subset X$ with finite measure and for every $\varepsilon>0$, there exists $A_{\varepsilon} \in \mathcal{A}_{F}$ such that $A_{\varepsilon} \subset A$ and $\mu\left(A \backslash A_{\varepsilon}\right)<\varepsilon$.

(b2) Let $A \in \mathcal{A}_{F}$ and let $K$ be a finite subset of $\Gamma$ such that

$$
\bigsqcup_{\delta \in F} A \delta \subset \bigsqcup_{\gamma \in K} \gamma Y
$$

For $\gamma \in \Gamma$, set, as in Definition 1.3.

$$
X_{A}(\gamma)=\left\{x \in A \cap \gamma^{-1} \cdot A: \alpha(\gamma, x) \in F\right\} .
$$

Then $X_{A}(\gamma)=\emptyset$ for every $\gamma \notin K K^{-1}$.

In particular, $\alpha$ satisfies condition (ii) of Definition 1.3 .

Proof. (a) Using induction on $|K|$, it suffices to prove the claim for singleton sets. Thus, let $A \subset X$ be a Borel set with finite measure, let $\gamma \in \Gamma$ and let $\varepsilon>0$. There exists a finite set $F \subset \Delta$ such that

$$
\sum_{\delta \notin F} \sigma\left(A \cap \gamma^{-1} X \delta\right)<\varepsilon .
$$

Then the Borel set $A_{\varepsilon}:=\bigsqcup_{\delta \in F} A \cap \gamma^{-1} X \delta$ is a subset of $A$ such that $\sigma\left(A \backslash A_{\varepsilon}\right)<\varepsilon$ and $\gamma A_{\varepsilon} \subset \bigsqcup_{\delta \in F} X \delta$.

(b1) Put $A F=\bigsqcup_{\delta \in F} A \delta$. Since $\sigma(A F)=|F| \sigma(A)=|F| \mu(A)<\infty$, there exists a finite set $K \subset \Gamma$ such that

$$
\sum_{\gamma \notin K} \sigma(A F \cap \gamma Y)<\varepsilon .
$$

Put $Z=A F \cap\left(\bigsqcup_{\gamma \in K} \gamma Y\right)$. For every $\delta \in F$, put $Z_{\delta}=\left(Z \delta^{-1}\right) \cap X$, so that $Z=\bigsqcup_{\delta \in F} Z_{\delta} \delta$. Finally, put

$$
A_{\varepsilon}=\bigcap_{\delta \in F} Z_{\delta}
$$

Then $A_{\varepsilon} \subset Z_{\delta}$ for every $\delta \in F$, hence $A_{\varepsilon} \delta \subset Z_{\delta} \delta \subset Z$ for every $\delta \in F$, so that $\bigsqcup_{\delta \in F} A_{\varepsilon} \delta \subset Z$. One has:

$$
\begin{aligned}
\mu\left(A \backslash A_{\varepsilon}\right) & =\sigma\left(A \backslash A_{\varepsilon}\right) \\
& =\sigma\left(A \cap\left(\bigcup_{\delta \in F} Z_{\delta}^{c}\right)\right) \\
& \leq \sum_{\delta \in F} \sigma\left(A \cap Z_{\delta}^{c}\right)=\sum_{\delta \in F} \sigma\left(A \delta \backslash Z_{\delta} \delta\right) \\
& =\sigma(A F \backslash Z)<\varepsilon .
\end{aligned}
$$

This ends the first part of the proof of the lemma.

(b2) Let $A$ and $K$ be as stated, and let $\gamma \in \Gamma$. If $X_{A}(\gamma)$ contains some element $x$, 
then $\alpha(\gamma, x) \in F$ and $\gamma x \in A F$. It follows that

$$
x \in\left(\bigsqcup_{\gamma^{\prime} \in K} \gamma^{\prime} Y\right) \cap\left(\bigsqcup_{\gamma^{\prime \prime} \in K} \gamma^{-1} \gamma^{\prime \prime} Y\right) .
$$

This implies that there are $\gamma^{\prime}, \gamma^{\prime \prime} \in K$ such that $\gamma=\gamma^{\prime \prime} \gamma^{\prime-1} \in K K^{-1}$.

\section{REFERENCES}

1. C. Anantharaman-Delaroche. Amenable correspondences and approximation properties for von Neumann algebras. Pac. J. Math. 171 (1995), 309-341.

2. P.-A. Cherix, M. Cowling, P. Jolissaint, P. Julg, and A. Valette. Groups with the Haagerup property (Gromov's a-T-menability). Birkhäuser, Basel, (2001).

3. M. COWling ANd U. HaAgerup. Completely bounded multipliers of the Fourier algebra of a simple Lie group of real rank one. Invent. Math. 96 (1989), 507-549.

4. A. Furman Gromov's measure equivalence and rigidity of higher rank lattices. Ann. Math. 150 (1999), 1059-1081.

5. A. Furman Orbit equivalence rigidity. Ann. Math. 150 (1999), 1083-1108.

6. F.P. Greenleaf. Amenable actions of locally compact groups. Journal Funct. Anal. 4 (1969) 295-315.

7. P. Jolissaint. Borel cocycles, approximation properties and relative property T. Ergod. Th. E Dynam. Syst. 20(2) (2000) 483-499.

8. P. Jolissaint. Invariant states and a conditional fixed point property for affine actions. Math. Ann. 304 (1996), 561-579.

9. S. KnudBy. The weak Haagerup property. To appear in Trans. Amer. Math. Soc. (2014), 41 pages.

10. W. Rudin. Real and Complex Analysis. McGraw-Hill Inc. New-York, (1987).

11. R.J. Zimmer. Amenable pairs of groups and ergodic actions and the associated von Neumann algebras. Trans. Amer. Math. Soc. 243 (1978), 271-286.

Current address: Université de Neuchâtel, Institut de Mathématiques, E.-Argand 11, CH-2000 Neuchâtel, Switzerland

E-mail address: paul.jolissaint@unine.ch 